\title{
Effects of Danqi Yishen Capsule on Blood Lipid Levels and Hemorheological Indicators of Hyperlipidemia Patients
}

\author{
Li Li ${ }^{*}$, Yan Li², Hui Li ${ }^{2}$ \\ ${ }^{1}$ Faculty of Pharmacy, Guangxi University of Chinese Medicine, Nanning, China \\ ${ }^{2}$ Shanxi Hospital of Traditional Chinese Medicine, Taiyuan, China \\ Email: *anceor2009@163.com
}

How to cite this paper: $\mathrm{Li}, \mathrm{L}$., $\mathrm{Li}, \mathrm{Y}$. and $\mathrm{Li}$ H. (2021) Effects of Danqi Yishen Capsule on Blood Lipid Levels and Hemorheological Indicators of Hyperlipidemia Patients. Chinese Medicine, 12, 19-28.

https://doi.org/10.4236/cm.2021.122003

Received: April 25, 2021

Accepted: June 6, 2021

Published: June 9, 2021

Copyright (c) 2021 by author(s) and Scientific Research Publishing Inc. This work is licensed under the Creative Commons Attribution International License (CC BY 4.0).

http://creativecommons.org/licenses/by/4.0/

\begin{abstract}
Objective: To explore the effects of Danqi Yishen Capsule (Danqi Capsule for Tonifying Kidney) on blood lipid levels and hemorheological indicators of hyperlipidemia patients. Methods: A total of 96 patients with hyperlipidemia were randomly divided into the control group and observation group of 48 cases respectively. The control group was given by simvastatin in oral administration of $10 \mathrm{mg}$ half an hour after dinner, once a day, while the observation group based on the treatment of the control group, was orally given Danshen Yishen Capsule of three capsules each time, three times a day. The treatment of both groups lasted for two months. Then the comparison focused on blood lipid levels, hemorheological indicators, and adverse reactions between both groups before and after the treatment. Results: There was no significant difference in blood lipid levels and hemorheological indicators between both groups before the treatment $(P>0.05)$. The symptoms of both groups were significantly improved after the treatment, and the difference before and after the treatment was statistically significant $(P<0.05)$, and the difference between both groups was also statistically significant $(P<0.05)$. There was no significant difference in the incidence rate of adverse reactions between both groups $(P>0.05)$. Conclusion: Danqi Yishen Capsule has a significant value in clinical applications, which can optimize clinical efficacy, improve blood lipid levels and hemorheological indicators of hyperlipidemia patients, as well as reduce the probability of adverse reactions in patients.
\end{abstract}

\section{Keywords}

Hyperlipidemia, Danqi Yishen Capsule (Danqi Capsule for Tonifying

Kidney), Blood Lipid Levels, Hemorheology, Adverse Reactions 


\section{Introduction}

Hyperlipidemia is a disease that the blood lipid level elevates due to abnormal lipid metabolism, often accompanied by hemorheological changes, which is an independent risk factor for atherosclerosis and especially one of the most common precipitating factors for coronary heart disease and cerebrovascular diseases [1]. Characterized by latency and slow progress, long-term hyperlipidemia can cause vascular endothelium dysfunction and lumen narrowing, which can affect blood flow and oxygen supply of local tissues, and finally lead to poor prognosis [2]. In clinical practice, medications such as statins are often applied for hyperlipidemia by lowering the blood lipid level, but it is easy to relapse after drug withdrawal and to induce toxic or side effects as gastrointestinal reactions [3]. In recent years, the author has applied Danqi Yishen Capsule on 48 cases of hyperlipidemia with a satisfactory curative effect. The research is reported as follows.

\section{Materials and Methods}

\subsection{Introduction}

A total of 100 cases were collected from patients complicated with hyperlipidemia and vascular disorders who had been admitted to the Department of Endocrinology of Shanxi Hospital of Traditional Chinese Medicine from July 2016 to July 2018. All the patients were randomly divided into control group and observation group of 50 cases respectively. A total of 96 patients successfully completed the research with the loss to follow-up of two cases in each group. The control group included 30 male patients and 18 female patients aged $56.61 \pm$ 5.42 on average, with the average course of disease of $15.78 \pm 4.75$ years. There were 29 male patients and 19 female patients aged $58.16 \pm 5.78$ on average, with the average course of disease of $15.20 \pm 5.32$ years in the observation group. There was no statistical difference in the baseline data above $(P>0.05)$, and the research had been approved by the ethics committee.

\subsection{Inclusion Criteria}

The subjects met the diagnostic criteria of hyperlipidemia in 2016 Chinese Guideline for the Management of Dyslipidemia in Adults [4]. Besides, they also conformed the diagnostic criteria of the pattern of qi deficiency and blood stasis in Guiding Principles for Clinical Research on Novel Chinese Materia Medica, which is manifested as shortage of qi, laziness to speak, limb weakness and numbness, sense of ant's marching on the skin, tingling sensation at the tip of fingers, pale tongue with thin and white coating, thready and weak pulse, as well as skin ecchymosis or petechiae [5]. Those symptoms above showed in 96 cases, and there was no significant difference in the conditions between both groups.

\subsection{Exclusion Criteria}

Those conditions had been excluded from the research, including severe liver 
and kidney diseases, systematic infection as well as drug allergy; severe mental illness and cognitive disorders; pregnancy and lactation; failure in efficacy or safety evaluation due to incomplete date resulting from patients' refusal to continue the treatment or non-cooperation, and a few cases of loss to follow-up.

\subsection{Therapeutic Methods}

Patients in both groups were required to quit smoking and alcohol as well as keep a strict control in diet and exercise. The oral administration of $20 \mathrm{mg}$ simvastatin tablets (Specification: $10 \mathrm{mg}$; Manufacturer: Beijing Front Pharmaceutical Co., Ltd.; SFDA approval No. H20030028) was given to the control group in the evening for 8 weeks straight. The observation group was additionally given Danqi Yishen Capsule (Specification: $0.5 \mathrm{~g} \times 40$ capsules per box; Shanxi Hospital of Traditional Chinese Medicine; Approval No. AZ20080218) orally of four capsules each time, three times a day for 8 weeks straight.

\subsection{Observation Index}

1) Based on the laboratory examination, a series of indicators of blood lipid and hemorheology before and after the treatment was compared in both groups, including triglyceride (TG), total cholesterol (TC), low density lipoprotein cholesterol (LDL-C), high density lipoprotein cholesterol (HDL-C), high-shear whole blood viscosity, low-shear whole blood viscosity, plasma viscosity, fibrinogen, etc.

2) There was a set of criteria for evaluating therapeutic effects [6]. After the treatment, the effective results showed when the level of TC, TG and LDL-C decreased more than $20 \%, 40 \%$ and $30 \%$ respectively while the level of HDL-C increased more than $0.26 \mathrm{mmol}$; the symptom improvement could be seen when the level of TC, TG and LDL-C decreased at the range of $10 \%$ to $20 \%, 20 \%$ to $40 \%$ and $15 \%$ to $30 \%$ respectively while the level of HDL-C increased at the range of 0.01 to $0.26 \mathrm{mmol}$; the treatment was invalid when the changes of TC, TG, LDL-C and HDL-C failed to meet the criteria above.

Total effective rate $=($ effective case + improved case $) /$ total case $\times 100 \%$.

3) The adverse reactions during the treatment of both groups were recorded, such as dry mouth, diarrhea, myalgia, abdominal distension, etc.

\subsection{Statistical Analysis}

The statistical software SPSS 19.0 was adopted to conduct data analysis and processing. The numeration data was represented by percentage (\%) and analyzed by chi-square test, and the measurement data was represented by mean \pm standard deviation $(\bar{x} \pm s)$. The independent sample t-test was applied for intergroup comparison, and the paired sample t-test was used for intragroup comparison, and $\mathrm{P}$-value higher than $0.05(P<0.05)$ indicates that the difference was statistically significant. 


\section{Results}

\subsection{Laboratory Test Results}

There was no significant difference in the levels of blood lipid and hemorheology between both groups before the treatment $(P>0.05)$. After the treatment, the levels of blood lipid and hemorheology in the observation group were significantly lower than those in the control group, and the difference between both groups had a statistical significance $(P<0.05)$, as Tables $1-4$ shows as follows.

\subsection{Comparisons of Clinical Efficacy}

After the treatment, the total effective rate of the observation group was $93.75 \%$, which was significantly higher than that of the control group manifested as $79.17 \%(P<0.05)$, as Table 5 shows as follows.

\subsection{Adverse Reactions}

There was no significant difference in incidence rates of adverse reactions between observation group and control group $(P>0.05)$, as Table 6 shows as follows.

Table 1. Changes of TG and TC before and after the treatment $(\mathrm{n}=48 \quad \bar{x} \pm s)$.

\begin{tabular}{|c|c|c|c|c|c|}
\hline \multirow{2}{*}{ Group } & \multirow{2}{*}{ Case number } & \multicolumn{2}{|c|}{$\mathrm{TG}(\mathrm{mmol} / \mathrm{L})$} & \multicolumn{2}{|c|}{$\mathrm{TC}(\mathrm{mmol} / \mathrm{L})$} \\
\hline & & Before the treatment & After the treatment & Before the treatment & After the treatment \\
\hline Observation group & 48 & $3.91 \pm 0.20$ & $1.65 \pm 0.03^{*}$ & $7.68 \pm 0.05$ & $4.73 \pm 0.12^{*}$ \\
\hline Control group & 48 & $3.94 \pm 0.18$ & $2.01 \pm 0.03^{*}$ & $7.67 \pm 0.11$ & $4.86 \pm 0.16^{*}$ \\
\hline
\end{tabular}

Note: Comparisons before and after the treatment in the same group, ${ }^{*} P<0.05$.

Table 2. Changes of LDL-C and HDL-C before and after the treatment $(\mathrm{n}=48 \quad \bar{x} \pm s)$.

\begin{tabular}{ccccc}
\hline \multirow{2}{*}{ Group } & Case number & \multicolumn{2}{c}{ HDL-C $(\mathrm{mmol} / \mathrm{L})$} & \multicolumn{2}{c}{ LDL-C (mmol/L) } & \multicolumn{2}{c}{ Before the treatment } & After the treatment \\
\cline { 3 - 4 } & & Before the treatment & After the treatment & $3.41 \pm 0.75$ \\
Observation group & 48 & $1.21 \pm 0.04$ & $1.31 \pm 0.03^{*}$ & $2.81 \pm 0.95^{*}$ \\
Control group & 48 & $1.23 \pm 0.02$ & $1.37 \pm 0.01^{*}$ & $3.48 \pm 0.95$ \\
\hline
\end{tabular}

Note: Comparisons before and after the treatment in the same group, ${ }^{*} P<0.05$.

Table 3. Changes of hemorheology before and after the treatment $(\mathrm{n}=48 \quad \bar{x} \pm s)$.

\begin{tabular}{|c|c|c|c|c|c|c|}
\hline \multirow{2}{*}{ Group } & \multicolumn{2}{|c|}{ High-shear whole blood viscosity (mPa.s) } & \multicolumn{2}{|c|}{ Low-shear whole blood viscosity $(\mathrm{mPa} \cdot \mathrm{s})$} & \multicolumn{2}{|c|}{ plasma viscosity $(\mathrm{mPa} \cdot \mathrm{s})$} \\
\hline & Before the treatment & After the treatment & Before the treatment & After the treatment & Before the treatm & fter the treatment \\
\hline Observation group & $6.72 \pm 1.05$ & $4.11 \pm 0.16^{* * \Delta}$ & 11.32 & $7.78 \pm 1.83^{* \Delta}$ & $1.59 \pm 0.46$ & $0.91 \pm 0.05^{\star_{\Delta}}$ \\
\hline Control group & $6.56 \pm 1.02$ & $5.03 \pm 1.03^{*}$ & $11.35 \pm 2.23$ & $9.25 \pm 1.05^{*}$ & $1.56 \pm 0.49$ & $1.35 \pm 0.29^{*}$ \\
\hline
\end{tabular}

Note: Comparisons before and after the treatment in the same group, ${ }^{*} P<0.05$; comparisons between both groups after the treatment, $\Delta P<0.05$.

Table 4. Changes of hemorheology before and after the treatment $(\bar{x} \pm s)$.

\begin{tabular}{|c|c|c|c|c|c|}
\hline \multirow{2}{*}{ Group } & \multirow{2}{*}{ Case number } & \multicolumn{2}{|c|}{ Hematocrit value (\%) } & \multicolumn{2}{|c|}{ Fibrinogen $(g / L)$} \\
\hline & & Before the treatment & After the treatment & Before the treatment & After the treatment \\
\hline Observation group & 48 & $0.65 \pm 0.11$ & $0.35 \pm 0.06^{*}$ & $4.29 \pm 0.56$ & $3.57 \pm 0.41^{* \Delta}$ \\
\hline Control group & 48 & $0.64 \pm 0.12$ & $0.53 \pm 0.03^{*}$ & $4.34 \pm 0.52$ & $4.01 \pm 0.64^{*}$ \\
\hline
\end{tabular}

Note: Comparisons before and after the treatment in the same group, ${ }^{*} P<0.05$; comparisons between both groups after the treatment, $\Delta P<0.05$. 
Table 5. Comparisons of clinical efficacy between both groups (n (\%)).

\begin{tabular}{cccccc}
\hline Group & Case number & $\begin{array}{c}\text { Effective rate } \\
\text { (case number) }\end{array}$ & $\begin{array}{c}\text { Improvement rate } \\
\text { (case number) }\end{array}$ & $\begin{array}{c}\text { Ineffective rate } \\
\text { (case number) }\end{array}$ & Total effective rate \\
\hline \multirow{2}{*}{ Observation group } & 48 & $26(54.16)$ & $19(39.58)$ & $3(6.25)$ & $45(93.75)^{*}$ \\
& $28.5 \%(14)$ & & $46.9 \%(23)$ & $24.4 \%(12)$ & $75.4 \%$ \\
Control group & 48 & $15(31.25)$ & $23(47.91)$ & $12(25)$ & $38(79.17)$ \\
& $28.5 \%(14)$ & & $46.9 \%(23)$ & $24.4 \%(12)$ & $75.4 \%$ \\
\hline
\end{tabular}

Table 6. Comparisons of incidence rates of adverse reactions between both groups ( $\mathrm{n}(\%)$ ).

\begin{tabular}{cccccccccc}
\hline \multirow{2}{*}{ Group } & \multirow{2}{*}{ Case number } & \multicolumn{2}{c}{ Dry mouth } & \multicolumn{2}{c}{ Muscular soreness } & \multicolumn{2}{c}{ Gastrointestinal reactions } & \multicolumn{2}{c}{ Incidence rates of adverse reactions } \\
\cline { 3 - 9 } & & Case number & $\%$ & Case number & $\%$ & Case number & $\%$ & $\%$ & $3(2.22)$ \\
Observation group & 48 & 1 & 2.13 & 1 & 2.13 & 1 & 2.13 & $10(2.63)$ \\
Control group & 48 & 3 & 2.22 & 3 & 2.22 & 4 & 2.27 & 10 \\
\hline
\end{tabular}

\section{Discussion}

Hyperlipidemia is a metabolic disease with a high incidence rate among the elderly [7] [8]. "Deficiency" is a physiological characteristic of the elderly, which is manifested as gradual exhaustion of yin and yang, qi and blood, zang-fu organs, physiological function as well as physical condition. The pathogenesis of geriatric diseases is due to kidney fire's failure in warming and spleen's loss in transportation and transformation [9]. The root cause of hyperlipidemia is lipid accumulation in the blood and vessels. According to TCM, hyperlipidemia is a kind of humoral metabolic disorder because the lipid originates from the body fluid, of which both occurrence and development have a close correlation with zang-fu organs related to the metabolism of body fluid [10]. Most TCM physicians believe that the pathogenesis of hyperlipidemia has a correlation with kidney deficiency based on its high incidence rate among the elderly [11] [12] [13] [14].

Modern researches have found that some people suffer from congenital gene defect of lipoprotein, lipoprotein receptor and lipase was due to heredity [15], which suggests that hyperlipidemia is closely related to kidney disorders, namely congenital deficiency. As Chapter 34 named Discussion on Disharmony of Plain Questions (Suwen) says, "The kidney is an organ of water and governs fluids." Kidney plays a key role in the formation, transportation, and transformation of body fluid. The deficiency of kidney yang can lead to disorders of qi transformation of body fluid. As a result, the fluid will develop into obesity by turning into phlegm, dampness, and lipid instead of transforming as energy [16] [17] [18]. For another, the accumulation of phlegm, dampness, or lipid, as a pathological product, can block the normal circulation of qi, and further lead to abnormal lipid metabolism so as to cause dyslipidemia [19]. Besides, the incidence rate of hyperlipidemia among the elderly has increased significantly. It especially occurs among postclimacteric female patients as well as middle-aged and older male patients, which has confirmed the theory of kidney deficiency in TCM [20]. Modern studies have verified that certain functions of kidney in TCM are similar 
to those of the hypothalamic-pituitary-target gland axis. Pituitary hormone of hypothalamus can regulate the homeostasis of the dynamic equilibrium of blood lipid, and thus the dysfunction of the hypothalamic-pituitary-target gland axis can lead to lipid metabolism disorders, which is also a modern explanation to the view that kidney deficiency can lead to hyperlipidemia [21]. To sum up, it can be concluded that the dysfunction of spleen and kidney as well as qi or yang deficiency of spleen and kidney, are the root cause of hyperlipidemia.

If the disease can't be detected and treated timely in the pathological stage manifested as dysfunctions of spleen and kidney, years later it will develop into blood stasis, or exogenous pathogenic factors still remain and lead to the exhaustion of qi and blood, which then turns into blood stasis and phlegm and finally blocks meridians and collaterals, according to Lin Zheng Zhi Nan Yi An (Guide to Clinical Practice with Medical Records). Blood stasis is the clinical manifestation of hyperlipidemia [22]. It has been verified in modern researches that acute cardiovascular diseases originate from latent pathogens in phlegm and blood stasis, which also has a close relationship with improvement or aggravation of diseases [23], therefore both phlegm and blood stasis are considered as important underlying pathological factors of the onset of hyperlipidemia [24]. The deficiency of spleen and kidney can lead to dysfunctions of other zang-fu organs. If qi fails to transform into fluids, the essence of water and food will turn into turbidity rather than "clear blood". As a result, the accumulation of lipid and phlegm blocks the vessels and finally leads to poor blood circulation by phlegm retention and blood stasis [25], which is similar to the process of glucotoxicity and lipotoxicity induced by insulin resistance, chronic inflammation, and oxidative stress in modern medicine [26] [27]. The reason why numerous TCM physicians have focused on this stage of the disease is that the clear pattern differentiation can be defined for its apparent symptoms [28] [29]. A long-term accumulation of lipid toxin can lead to the deficiency of collaterals and vessels. Then lipid toxin will invade the body, spread along with collaterals and vessels, further aggravate the progress of disease and even develop into other disorders. A number of animal experiments [30] [31] and clinical trials [32] [33] have confirmed that the formula of promoting qi and blood can effectively improve dyslipidemia with anti-coagulation. Thus it can be seen that phlegm and blood stasis are not only pathological products but also pathogenic factors for aggravation of the disease during the occurrence and development of hyperlipidemia, and especially can aggravate and accelerate the progress of vascular complications.

There are several ingredients functioning varies as monarch, minister, assistant and envoy in Danqi Yishen Capsule. The monarch medicine includes Huangqi (Milkvetch Root; Radix Astragali Seu Hedysari), Taizishen (Heterophylly Falsestarwort Root; Radix Pseudostellariae) and Danshen (Danshen Root; Radix Salviae Miltiorrhizae), which can promote qi transformation so as to achieve the balance of energy metabolism. The minister medicine includes Dihuang (Prepared Rehmannia Root; Radix Rehmanniae Preparata), Baizhu (Largehead 
Atractylodes Rhizoma; Rhizoma Atractylodis Macro), Yiyiren (Coix Seed; Semen Coicis), Danggui (Chinese Angelica; Radix Angelicae Sinensis) and Gegen (Kudzuvine Root; Radix Puerariae), which can strengthen spleen and regulate liver, clear heat and resolve phlegm, as well as activate blood. The assistant and envoy medicines include Dahuang (Rhubarb Root and Rhizome; Radix Et Rhizoma Rhei), Qianshi (Gordon Euryale Seed; Semen Euryales), Jinyingzi (Cherokee Rose Fruit; Fructus Rosae Laevigatae) and Yinyanghuo (Epimedium Herb; Herba Epimedii) [34]. Before the treatment, there was a significant abnormality showed in the level of whole blood viscosity of patients in both groups. It indicated that the patient's blood was in a hypercoagulable state with slow blood flows and tissue hypoxia during the progression of the disease, which was consistent with the pathological process of hyperlipidemia. In the research, the levels of blood lipid and hemorheology of both groups were significantly improved after the treatment compared with those before the treatment, and the indicators above of the observation group were better than those of the control group, and the difference between both groups was statistically significant $(P<0.05)$. In addition to symptom improvement, the treatment was also beneficial to a better prognosis of patients. The results indicated that the treatment of western medicine combined with Danqi Yishen Capsule could enhance western medicine' effects on regulating lipid, effectively reduce the blood viscosity, improve hemorheology and microcirculation, elevate the level of plasma HDL-C, promote the transfer of LDL-C, regulate the function of body's immune system, as well as protect the cerebro-cardiovascular endothelial cells. Besides, the difference was no statistically significant in the incidence rate of adverse reactions between both groups $(P>0.05)$, which further indicated that Danqi Yishen Capsule had a safe and reliable effect by supporting western medicines on lipid regulation.

Due to its various severity, hyperlipidemia sometimes even has no apparent symptoms, and sometimes it is presented as a complex of deficiency, excess, cold and heat, or as a principle syndrome with several accompanied syndromes in different stages of the disease. However, the pattern differentiation and treatment of TCM relies on individual experience without a uniform pattern, and it is therefore significant and necessary to correlate the laboratory index with the pathological progress of hyperlipidemia. This paper, taking Chinese herbal compounds with Huangqi (Milkvetch Root; Radix Astragali Seu Hedysari) as a sample, pointed out that deficiency was the key to the onset of hyperlipidemia from the perspectives of both molecular biology and clinical efficacy on the basis of previous laboratory results [35] and clinical data. The turbidity often first occurs and then leads to various pathological products as phlegm, blood stasis, toxic substance, etc., which thus forms a pathological process of chain reaction. The onset of hyperlipidemia should be considered as a longitudinal and dynamic process in clinical treatment. Regardless of the types and stages of hyperlipidemia, the strengthening of spleen and stomach should be taken as the priority. In addition, it is essential to pay attention to both pathogenesis and results of the 
disease, and its treatment should focus on prevention, so as to put the idea of preventive treatment of diseases into TCM clinical practice.

\section{Foundation Project}

Doctoral Scientific Research Foundation Project of Guangxi University of Chinese Medicine (No. 2018BS021).

1) Guangxi University of Chinese Medicine, Faculty of Pharmacy, Nanning, 530020 .

2) Tianjin University of Traditional Chinese Medicine, Institute of Traditional Chinese Medicine, Tianjin, 301617.

\section{Conflicts of Interest}

The authors declare no conflicts of interest regarding the publication of this paper.

\section{References}

[1] Liu, X.J., Chen, C.F. and Liu, S.H. (2017) Clinical Observation of Coronary Heart Disease with Qi-Yin Deficiency and Blood Stasis Syndrome by Using Shengmai Powder Combined with Xuefu Zhuyu Decoction. Journal of Hunan University of Chinese Medicine, 37, 423-425.

[2] Wang, B. (2016) Effects of Xuefu Zhuyu Decoction on Prostacyclin and Thromboxane A2 in Blood Lipid and Plasma of Atherosclerosis Rats. Journal of Community Medicine, 14, 57-58.

[3] Ma, L.Y. (2018) Research Progress on Related Adverse Reactions and Non-Cardiovascular Effects of Statins. Guide of China Medicine, 16, 8-9.

[4] Zhu, J.R., Gao, R.L., Zhao, S.P., et al. (2017) 2016 Chinese Guideline for the Management of Dyslipidemia in Adults. Chinese Journal of Health Management, 11, 7-28.

[5] Zheng, X.Y. (2002) Guiding Principles for Clinical Research on Novel Chinese Materia Medica. China Medical Science Press, Beijing, 85-89.

[6] Zhang, S.H., Wei, G. and Xu, J.W. (2019) To Explore the Clinical Efficacy and Safety of Different Doses of Simvastain Treatment of Elderly Hypertension Combined Hyperlipidemia. Journal of Frontiers of Medicine, 9, 112-113.

[7] Yu, X.M., Yang, G.H. and Li, P. (2014) Mechanism of Lowering Blood Lipids of Xuefuzhuyu Decoction in Patients with Hyperlipidemia. Liaoning Journal of Traditional Chinese Medicine, 41, 289-291.

[8] Wu, X., Zhu, B., Li, W.X., et al. (2013) Effect of Xuefu Zhuyu Decoction on Serum Adiponectin Level in Hyperlipidemia SD Rats. Progress in Veterinary Medicine, 34, 76-79.

[9] Song, X. (2008) Physiological Characteristics of the Elderly in Traditional Chinese Medicine. Journal of Chengdu University of Traditional Chinese Medicine, 31, 13-16.

[10] Cui, L.L. (2012) The Damage of Hyperlipidemia and Its Prevention with TCM. Western Journal of Traditional Chinese Medicine, 25, 101-102.

[11] Ye, Q. and Gu, Y.H. (2014) Introduction of Chinese Medicine Treatment of Hyperlipemia. Journal of Liaoning University of Traditional Chinese Medicine, 16, 159-160. 
[12] Yili, G. (2016) Research Progress of Traditional Chinese Medicine on Hyperlipidemia. Guangming Journal of Chinese Medicine, 31, 444-447.

[13] Gong, Y.P. and Song, G.P. (2001) Correlations between Dysfunctions of Zang-Fu Organs and Hyperlipidemia. Chinese Journal of Information on Traditional Chinese Medicine, 8, 7-8.

[14] Li, T. and Zhao, Y.Q. (2013) Discussion on Hyperlipidemia Based on the Perspective of Kidney Blood. Clinical Journal of Chinese Medicine, 5, 58-59.

[15] Ford, E.S., Giles, W.H. and Dietz, W.H. (2002) Prevalence of the Metabolic Syndrome among US Adults: Findings from the Third National Health and Nutrition Examination Survey. JAMA, 287, 356-359. https://doi.org/10.1001/jama.287.3.356

[16] Xu, Y.F., Liu, J. and Li, Z.H. (2011) Professor LI Zhen-Hua's Experience in Treating Obesity. China Journal of Traditional Chinese Medicine and Pharmacy, 26, 15421543.

[17] Chen, Q.G., Dai, Z.Q., Tao, F., et al. (2016) Ding Xueping's Clinical Experience in Treating Obesity. Shanghai Journal of Traditional Chinese Medicine, 50, 17-19.

[18] Xu, S.S., Gong, M.R. and Sun, Y.N. (2015) Simple Obesity Treatment from Spleen and Stomach. Liaoning Journal of Traditional Chinese Medicine, 42, 628-629.

[19] Feng, X.R. (2007) Analysis on "Kidney Is the Resource of Phlegm". Lishizhen Medicine and Materia Medica Research, 18, 979.

[20] Wang, A.L., Xu, H. (2015) Professor Xu Hao's Clinical Experience in Treating Hyperlipidemia Based on Constitution Differentiation from Liver, Spleen and Kidney. China Journal of Traditional Chinese Medicine and Pharmacy, 30, 1999-2001.

[21] Zhang, J.M., La, W.Y. (2008) Research Progress of the Treatment of Traditional Chinese Medicine on Hyperlipidemia of Type 2 Diabetes. Hebei Journal of Traditional Chinese Medicine, 30, 214-216.

[22] Yang, F., Huang, J.R. and Xiang, N. (2009) Relations between Dysfunctions of Liver and Spleen and Insulin Resistance. Lishizhen Medicine and Materia Medica Research, 20, 1556-1557.

[23] Ren, J.X. (2003) Exploration on Latent Pathogens II: Miscellaneous Diseases. Chinese Medicine Modern Distance Education of China, 1, 8.

[24] Mou, Z.Y. (2015) Correlations between Acute Coronary Syndrome and Latent Pathogens in Phlegm and Stasis. Chinese Journal of Gerontology, 35, 294-296.

[25] Chen, W.K. (1988) Prevention and Treatment of Traditional Chinese Medicine on Hyperlipidemia. Traditional Chinese Medicine Ancient Books Publishing House, Beijing, 13.

[26] Li, M.Q., Lin, Y., Xiang, L., et al. (2019) Dan-Zhuo and Glucolipid Metabolic Disorders. World Chinese Medicine, 14, 652-655.

[27] He, W., Xing, Y.R., Zhang, J.M., et al. (2013) Analysis and Summary of Modern TCM Turbid Pathogenic Factor Theory. Shaanxi University of Chinese Medicine, 36, 13-16.

[28] Yin, F., Chen, X.Z. and Yang, L. (2006) Pattern Differentiation and Treatment on Hyperlipidemia Based on Kidney Deficiency and Blood Stasis. Journal of Sichuan of Traditional Chinese Medicine, 24, 25-26.

[29] Du, S., Hu, J.Q., Lu, H.R. (2015) Review on Modern Theoretical Research Progress of Syndrome of Intermingled Phlegm and Blood Stasis. Chinese Journal of Basic Medicine in Traditional Chinese Medicine, No. 4, 477-482.

[30] Tian, Y., Xu, Y., Xia, L.N., et al. (2014) Effects of Shenqi Compound on Serum IL-6 
and Blood Lipid in High-fat Feeding GK Rats. Forum on Traditional Chinese Medicine, 29, 43-44.

[31] Xu, H. (2017) The Effect of Compound Danshen Dripping Pills and Shenqiyiqi Dripping Pills on Cardiovascular Diseases. Shandong University, Jinan.

[32] Zhao, X.J. (2018) Effect Observation of Shenqi Compound Combined with Vildagliptin on Diabetic Macrovascular Complications. Modern Journal of Integrated Traditional Chinese and Western Medicine, 27, 2804-2807.

[33] Yan, J. and Jia, H.L. (2019) Effects of Xuefu Zhuyu Decoction on Blood Lipid Levels and Hemorheological Indicators of the Patients with Hyperlipemia. Western Journal of Traditional Chinese Medicine, 32, 47-49.

[34] Liu, D.W. (2018) Regulation of Danqi Yishen Capsule on Diabetic Nephropathy and Laboratory Indicators. Chinese Journal of Primary Medicine and Pharmacy, 25, 3141-3144.

[35] Li, L. and Wang, T. (2020) Absorbed Components of Jinqi Jiangtang Formula and Its Effects on Lipid Accumulation of HepG2 Cells. Chinese Remedies \& Clinics, 20, 356-358. 